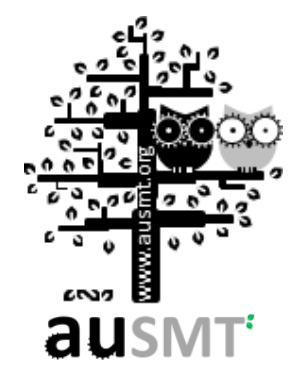

\title{
High Efficient Synchronization-on-demand Protocol of IEEE 802.15.4 Wireless Sensor Network for Construction Monitoring
}

\author{
Ji-De Huang ${ }^{1}$, Wen-Jong $\mathrm{Wu}^{2}$, , and Chih-Ting Lin ${ }^{3, *}$ \\ ${ }_{1}^{1}$ Institute of Applied Mechanics, National Taiwan University, Taiwan \\ ${ }^{2}$ Department of Engineering Science and Ocean Engineering, National Taiwan University, Taiwan \\ ${ }^{3}$ Graduate Institute of Electronics Engineering, National Taiwan University, Taiwan \\ (Received 13 July 2011; Accepted 11 December 2011; Published on line 1 June 2012) \\ *Corresponding author: Chih-Ting Lin timlin@cc.ee.ntu.edu.tw; Wen-Jong Wu wjwu@ntu.edu.tw \\ DOI: $10.5875 /$ ausmt.v2i2.98
}

\begin{abstract}
Wireless Sensor Network (WSN) technology is currently being employed in the field of civil engineering, especially in the areas of structural monitoring and construction safety. When used in construction site safety, time-synchronization within the sensor network is required to obtain important correlations between events, such as the prevention of equipment collisions. Here, we develop a highly efficient time synchronization method for an ultra-precise localization system to prevent collision accidents at construction sites. Without interfering with the operation of the entire sensor network, the developed protocol can locally synchronize several nodes within 10 ms. By measuring the time uncertainty information through the time stamping, wireless sensor nodes can be synchronized with only two transmitted packages from the beacon or specific nodes. Based on this proposed approach, 0.8 micro-second synchronization error was experimentally achieved on an IEEE 802.15.4 platform. Employing the developed method, the localization system was implemented and experimentally examined to have accuracy within several centimeters in the range of $2 \mathrm{~m}$. This localization system can be used to identify the location of different construction equipment. This work demonstrates a simple and efficient approach to obtain high accuracy, low communication overhead, and robust synchronization for most WSN platforms to be used in automated monitoring at construction sites.
\end{abstract}

Keywords: Wireless sensor network; synchronization; ultrasonic localization; Anti-collision system

\section{Introduction}

Because of dynamical and complicated characteristics of civil construction sites, workers suffer from potential collisions with robotic equipment [1-3]. To improve the safety of construction sites, strict regulations for field operations are required at construction sites. However, unpredictable accidents still occur due to human error or carelessness. Subsequently, several methods involving technology have been proposed to prevent these accidents [2, 4]. Among these technologies, the wireless sensor network (WSN) has drawn attention due to its low-cost, flexibility, and ease of implementation at construction sites [5].

WSNs have become an emerging research field due to their promising potential in different applications and the advancement of integrated circuit technologies [6]. A WSN consists of hundreds of wireless communication nodes with sensing capabilities to collect the desired data. To obtain consistency and efficiency between nodes, coordinated interaction within the network is necessary [7]. In coordinating sensor node activities, time synchronization is one of the most important features to consider in a WSN system. Due to resource limitations and decentralized characteristics of WSNs, however, time 
synchronization consideration in a WSN is different that of wired communication systems [8, 9]. As a result, several protocols for time synchronization, e.g., reference broadcast synchronization (RBS), timing-sync mechanism for sensor networks (TPSN), and flooding time synchronization protocol (FTSP), have been proposed and implemented to overcome limitations posed by limited resources in each node [10,11].

Most WSN systems have employed IEEE 802.15.4 as the standard for their physical (PHY) layer and media access control (MAC) layer because of its lower consumption of power. It should be noted that IEEE 802.15.4 is implemented with random back-off of a carrier sense multiple access with collision avoidance (CSMA/CA) mechanism to enhance throughput and avoid interference. Although CSMA/CA promotes wireless communication, its random delays result in complicated time uncertainty in time synchronization. Consequently, previous synchronization protocols utilized a great deal of message exchanging to estimate the clock offset and uncertainties [12]. This, however, leads to network instability, especially in large-scale fields such as civil construction sites. To avoid collision accidents at such locations, it is important to have the capability of local, real-time identification without a full network check. Therefore, the development of a new protocol with fast synchronization capabilities and less interference with a large network is necessary.

In this work, we propose the implementation of the synchronization-on-demand (SOD) protocol for an IEEE 802.15.4 network. With two data transmissions without statistical estimation, the proposed synchronization is achieved with microsecond precision and minimized resource consumption. Through doing so, accidental collisions at construction sites can be avoided by using this localized protocol to trace the objects. In our evaluation of the functionality of the proposed protocol, the ultrasonic localization system was experimentally demonstrated to have the capability of locating objects within several centimeters.

Ji-De Huang received his M.S. degree from Institute of Applied Mechanics, National Taiwan University. He is currently Ph.D. Candidate of Institute of Applied Mechanics in National Taiwan University. His current research interests include wireless sensor network and embedded systems.

Wen-Jong Wu received his M.S and Ph.D. degrees from the Institute of Applied Mechanics, National Taiwan University in 1998 and 2003 respectively. Since 2003, he has been an Assistant Professor at the Dept. of Engineering Sience \& Ocean Engineering, National Taiwan University. His research interests include systems design, integration of precision metrology, smart sensor networks, and piezoelectric power devices.

Chih-Ting Lin received his Ph.D. degree from Department of Electrical Engineering and Computer Science of University of Michigan. He is currently Assistant Professor of Graduate Institute of Electronics Engineering in National Taiwan University. His current research interests include biosensors and micro devices.

\section{Synchronization-On-Demand (SOD) Protocol}

\subsection{Source of Time Uncertainty in WSN}

To obtain highly accurate synchronization within a WSN, it is important to understand the source of uncertainties in the process of radio-frequency (RF) transmission. Among the different categories of time delay factors [13] in RF transmission, handling interruption time, sending time, receiving time, and access time are unpredictable and are the major uncertainties which should be handled in the synchronization process. Handling interruption time is the duration between the microcontroller being interrupted from the MAC layer and being able to start dealing with packages. Sending time is the time required to setup and transfer packages to the MAC layer in the transmission node. Receiving time is the time needed for handling incoming packages in the receiving node. Access time is the time of waiting for the RF channel to become clear for transmission. Among these four factors, all but access time are highly dependent on the package content. In addition, handling interruption time is also dependent on the loading of the microcontroller. Access time depends on the standard specifying the physical and MAC layers for wireless transmissions. It should be noted that RF transmission is relatively small compared to other factors because the speed of electromagnetic wave propagation is much larger than the transmission distance. Therefore, RF transmission is not considered in this work. For WSNs based on the IEEE 802.15.4 standard, access time is mainly affected by the uncertain duration of random back-off due to CSMA/CA. This time uncertainty dramatically affects the precision of time synchronization.

\subsection{Design of SOD Protocol}

Instead of making error estimations, the major design concept of SOD is measuring the time uncertainty information through time-stamping during synchronization. Utilizing this method, nodes can be synchronized with only two transmitted packages from the beacon or specific nodes. Since it can be achieved within an extremely short time, it can re-synchronize as requested. Therefore, SOD can be implemented without considering clock drifts or skews of the local timer.

In implementing the developed SOD protocol, we assume the crystal oscillator of the beacon node and the receiving node run at stable frequencies. The beacon node sends two packages - synchronization and correction - to the receiving node. The synchronization package contains the time stamp of the beacon node. 


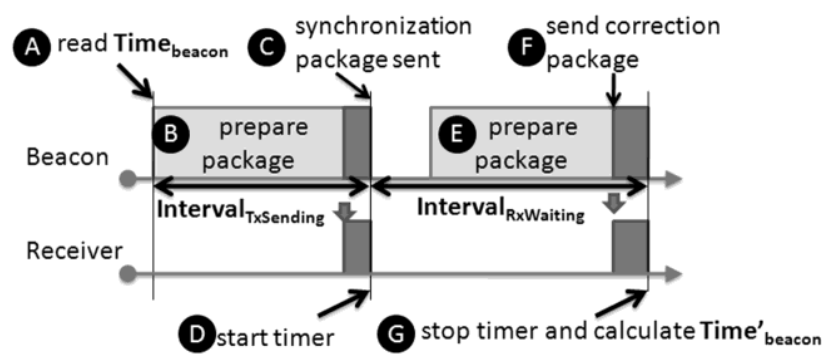

Figure 1. The schematic of the protocol sequence.

The correction package contains the uncertainty information. As shown in Figure 1, the detailed time diagram for the synchronization procedure can be described as follows: $(A)$ the beacon node reads its time stamp; (B) the time stamp is inserted into the synchronization package; (C) the beacon records another time stamp right after sending the synchronization package, and the Interval ${ }_{\text {TxSending }}$ can be obtained; (D) the receiver node records its time stamp after receiving package; (E) the Interval TxSending $_{\text {is inserted into the }}$ correction package; $(F)$ the beacon node sends the correction package; $(\mathrm{G})$ the receiver node picks up the correction package and records another time stamp to

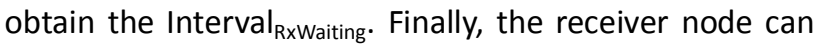
calculate the current time stamp of the beacon node as:

$$
\begin{aligned}
\text { Time }_{\text {receiver }}= & \text { Time }_{\text {beacon }}^{\prime} \\
= & \text { Time }_{\text {beacon }}+\text { Interval } \\
& + \text { Intervanding }_{\text {RxWaiting }}+\text { const. }
\end{aligned}
$$

where const. represents the predictable time errors such as computation time.

Since the microcontroller can record transmission duration, the previously unpredictable time delays, namely Sending Time. Access Time, and Receiving Time can be obtained. Additionally, Handling Interruption Time can be eliminated by firmware coding, such as setting the microcontroller into an idle state before any transmission during the synchronization procedure. As such, most of the unpredictable time delay sources can be identified through the developed SOD protocol. It should be noted that the time stamp exchange is not necessarily required in the SOD protocol, which can also be implemented by broadcasting. Moreover, the SOD protocol can achieve synchronization in a much faster fashion than other statistical-based time synchronization protocols. In addition, the multi-hops synchronization can be achieved by repeating the same procedure to nearby nodes in different regions.

The SOD has several important features that make it suitable for low power WSNs. The highly precise clock is only kept at the local beacon. All the other nodes can be aligned up through SOD protocol. Additionally, it is not necessary for nodes to keep a local hardware clock, e.g. crystal oscillator, as statistical methods do. As the precise synchronization is not needed, the nodes can also change to low frequency mode to save power. Furthermore, message exchange and iteration are not required in SOD, differing from traditional synchronization methods. Moreover, SOD can be applied in CSMA/CA enabled platforms like Zigbee, or other wireless networks with large access uncertainty.

\subsection{Error Source of SOD}

The developed SOD method measures each time interval directly with a hardware timer. Most of the time uncertainties can be eliminated except for clock jitter. The clock jitter error can be determined from one of three error sources. (A) Cycle $e_{R F}$ : jitters between received RF signal input and decoded digital signal output in the RF transceiver chip. This jitter accounts for one clock cycle of the output block in the RF transceiver chip. (B)

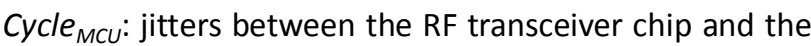
microcontroller chip. This jitter accounts for one clock cycle in the microcontroller. (C) Jitters between different nodes. This jitter accounts for the sum of $(A)$ and $(B)$ over both nodes. Based on the above, the jitter error between nodes in the same synchronization region can be determined as $4\left(\mathrm{Cycle}_{M C U}+\mathrm{Cycle}_{\mathrm{RF}}\right)$. Moreover, in multi-hop topography, the jitter error across synchronization regions can be expressed as $(2+4(n-1))$ $\left(\right.$ Cycle $_{M C U}+$ Cycle $\left._{R F}\right)$, where $n$ is the number of synchronization regions. It should be noted that the jitter error is in Gaussion distribution, which means it can be estimated by Kalman filter.

\subsection{SOD Experimental Verification}

To evaluate the proposed SOD protocol performance and demonstrate its capabilities, we implemented the protocol on a low-cost WSN platform for both beacon nodes and receiving nodes. The platform uses UZ2400 as its RF transceiver and MSP430F1611 as its microcontroller, as shown in Figures 2(A) and 2(B), respectively. UZ2400 is an IEEE 802.15.4 compatible RF transceiver $[14,15]$. It is capable of notifying the microcontroller when TX/RX is completed. In addition, MSP430F1611 shares the $5 \mathrm{MHz}$ clock generated from UZ2400. Therefore, there is no jitter between these two chips within the platform, meaning the jitter of $\mathrm{Cycle}_{\mathrm{MCU}}$ is zero. 
(A)

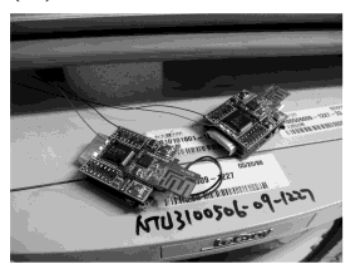

(B)

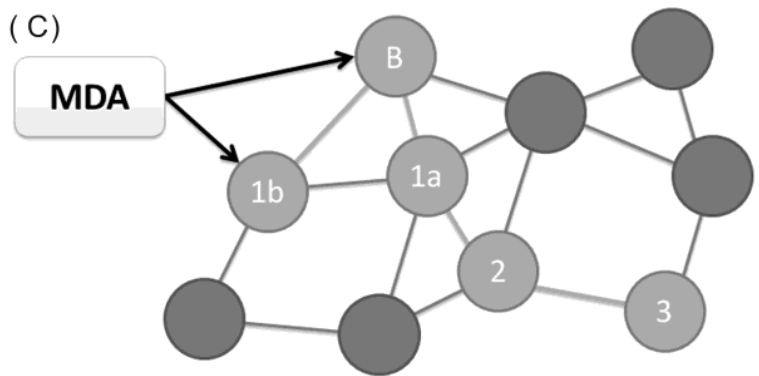

Figure 2. (A) The photo of super nodes; (B) The schematic of the SOD experimental setup for verifications; (C) The schematic of the experimental topography.
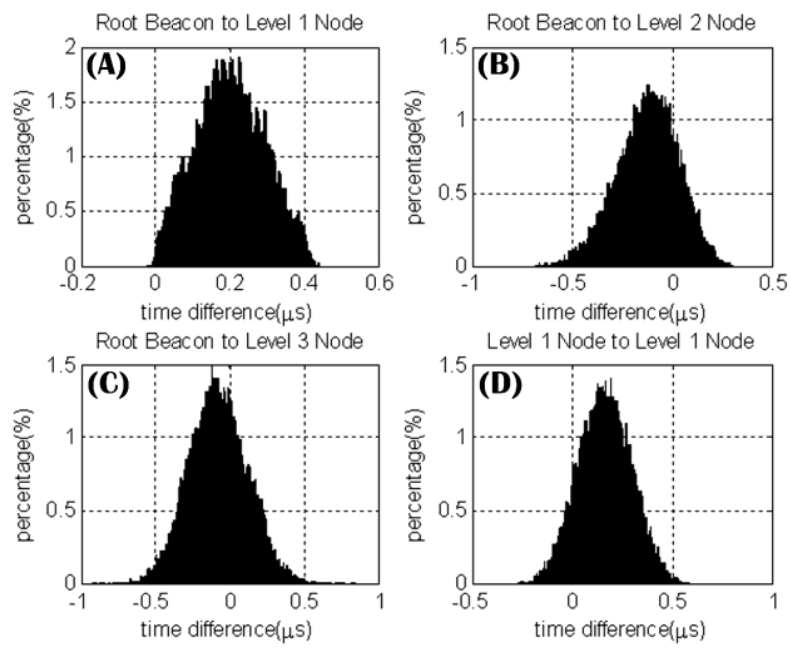

Figure 3. The quantitatively time synchronized results of SOD obtained by Modulation Domain Analyzer. (A) The beacon and nodes in one-hop range (B) The beacon and nodes in two-hop range (C) The beacon and nodes in three-hop range (D) The nodes within one hop. This statistical result demonstrates the synchronization error from single hop to triple hops.

The experimental network topography is shown in Figure 2(C). In this network topography, we adopted three layers to examine the performance of SOD in multi-hop and local synchronization scenarios. Furthermore, we enabled the CSMA/CA mechanism and followed the IEEE 802.15.4 standard during the experiments to mimic the real application scenario. In this topography, after synchronization the microcontroller of each node sends the pulse according to the synchronized global time. To directly identify the synchronized error, HP53310A Modulation Domain Analyzer (MDA) is used to analyze the time difference between pulses sent by different nodes. The histogram of synchronization error can be recorded by MDA and is shown in Figure 3.
In the experiments with 20,000 measurements for each node, the average synchronization error between the beacon and nodes in one hop was $0.2 \mu \mathrm{s}$ with standard deviation of $0.09 \mu \mathrm{s}$, resulting from the fundamental clock offset of the $5 \mathrm{MHz}$ crystal. For the nodes in two hops, the mean synchronization error to the beacon was $-0.12 \mu$ s with standard deviation of 0.15 $\mu \mathrm{s}$. For the nodes in three hops, the mean synchronization error to the beacon was $-0.17 \mu$ s with standard deviation of $0.2 \mu \mathrm{s}$. Based on these results, the local synchronization precision of the developed SOD protocol can be experimentally determined as $\pm 0.875 \mu \mathrm{s}$ within three hops, and the error propagation can be experimentally identified as $0.05 \mu$ s per hop. This experimental outcome clearly demonstrates that high accuracy can be achieved by SOD without any statistical techniques.

Compared to previous synchronized protocols such as TPSN and FTSP, the experimental evaluation of SOD also demonstrates higher synchronized accuracy. In detail, TPSN and FTSP have the average synchronization error of $17.61 \mu \mathrm{s}$ [14] and $1.48 \mu \mathrm{s}$ [13], respectively, within a single hop. It is clear that SOD is superior to TPSN. On the other hand, FTSP has similar synchronized error to SOD. However, it should be noted that FTSP and TPSN employ the special function of generating interruption at the start of frame delimiter (SFD) to achieve the elimination of the random back-off effect introduced by CSMA/CA. To our knowledge, only CC2520, CC2420, CC2430, and CC2431 can fulfill this required function for FTSP and TPSN among all RF transceivers following IEEE 802.15.4. In contrast, SOD extracts the access time uncertainty caused by CSMA/CA without using the specific SFD function. As a result, SOD can be implemented into various WSN platforms without this limitation.

\section{Ultrasonic Localization System}

\subsection{D Distance Measurement}

The major advantage of the developed SOD is that it can be achieved by several sensor nodes without global synchronization in the whole network. This dramatically improves the network stability and reduces communication latency in the WSN system. To experimentally demonstrate the collision-prevention potential of the developed SOD, an ultrasonic ranging system was implemented to measure the distance between two objects. For lower cost and ease of implementation, the ultrasonic ranging system utilized the time-of-flight (TOF) method to obtain the distance information $[13,16]$. In brief, the TOF method is achieved 
through using two ultrasonic transducers, i.e. sensor nodes, with one being the transmitter and the other being the receiver. The transmitter sends out modulated ultrasonic waves that induce the resonant vibration of the transducer in the receiver. The time difference between transmission and receiving, obtained by comparing the time stamps from two synchronized ultrasonic sensor nodes, can be used to estimate the distance between two transducers. Thus, it is critical to have highly precise synchronization.

In this ultrasonic ranging system, a $40 \mathrm{kHz}$ transducer is used to transmit/receive ultrasonic waves.
To quantitatively demonstrate the distance measurement by the developed SOD, only a single level synchronization is processed without lost of generalization. The experimental result of the 1-D distance measurement is shown in Figure 4 where the calculated distance and error with regard to real distance are shown. Each point stands for error between calculated and real distances. In general, the error was within $1 \mathrm{~mm}$ at a distance of $2 \mathrm{~m}$. In other words, the overall standard deviation of synchronization is within $1 \mu \mathrm{s}$. This result also shows great reproducibility of this ultrasonic distance measurement system.

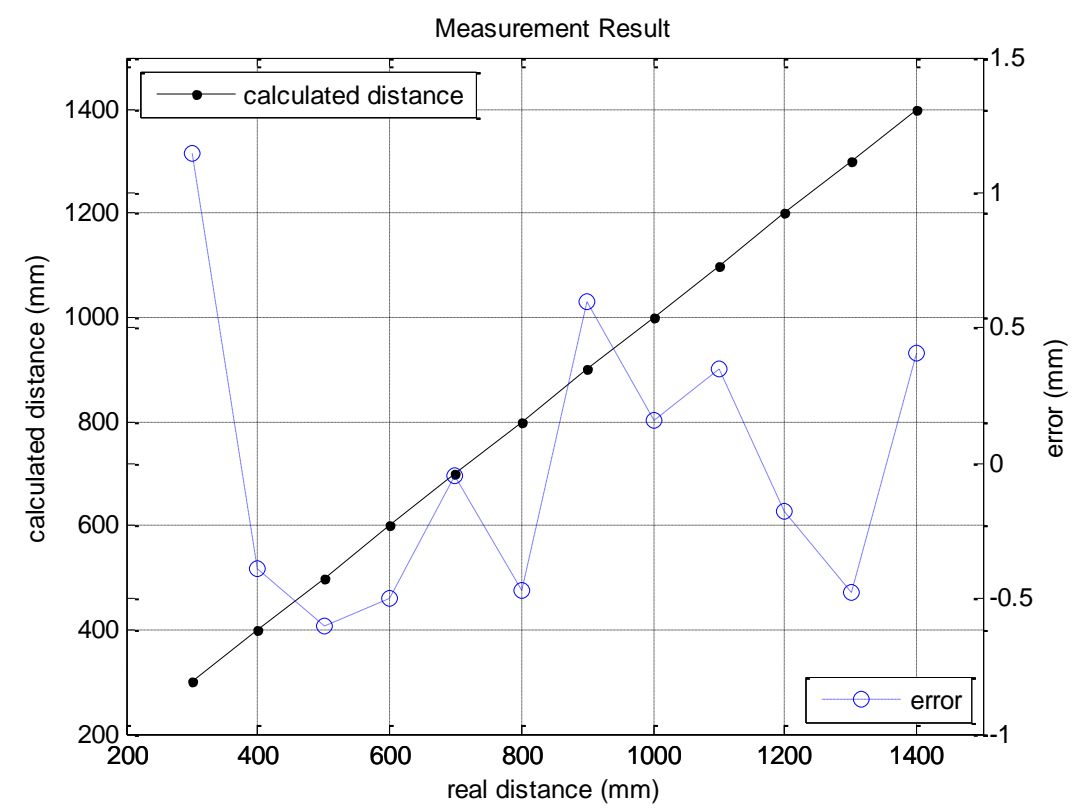

Figure 4. The experimental result of 1-D distance measurement.

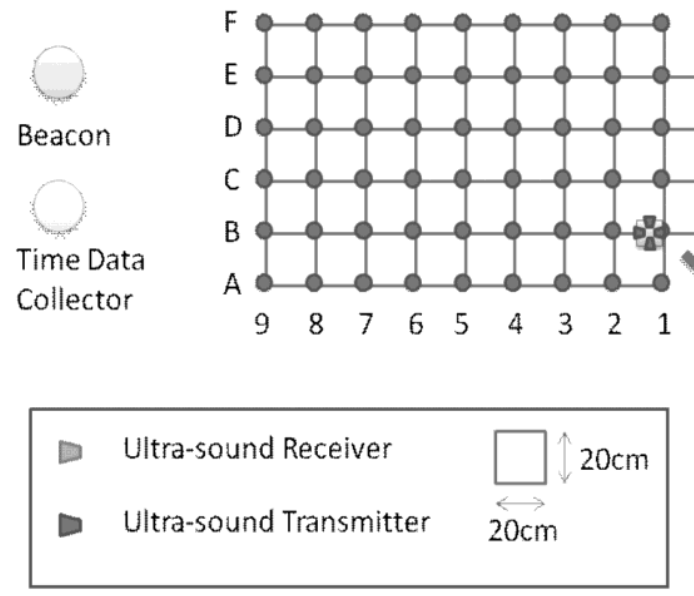
Sender Node

Receive Sensor Node:S1

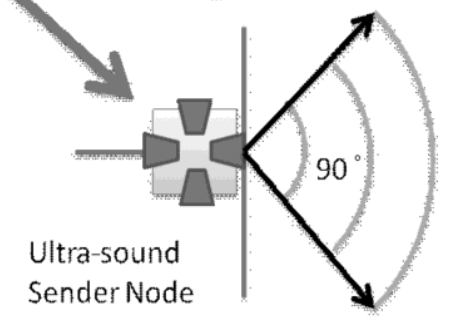

Figure 5. The experimental setup of 2D distance measurement. 


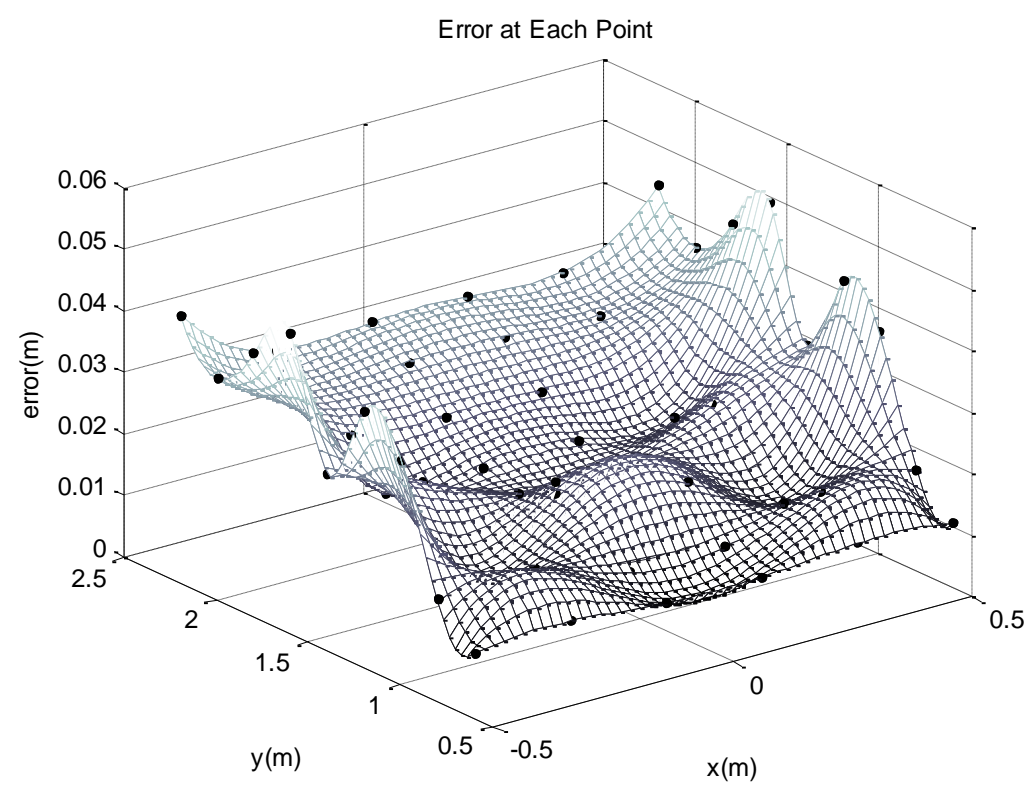

Figure 6. The experimental error of each location in $2 \mathrm{D}$ location measurement.

\subsection{D Location Measurement}

To mimic the monitoring of the operator of a piece of equipment during operation, the ultrasonic system was reconfigured to process $2 \mathrm{D}$ location measurement. In brief, the receiver nodes represent the tail of the equipment and the ultrasonic sensor node represents another object or worker. As shown in the system diagram in Figure 5, the beacon node sends time synchronization packages every $100 \mathrm{~ms}$. The ultrasonic sender node turns on an ultrasonic driver for $100 \mu \mathrm{s}$ as synchronized with the beacon node. Because the directivity of ultrasonic transducer is about $\pm 45^{\circ}$, four transducers are arranged to obtain full angle range. After being synchronized with the beacon node, the receiving sensor node listens for the ultrasonic signal, upon which it calculates the time difference and sends data to the time data collector, which then transmits data to the computer for analysis. During the experiment, the ultrasonic sender node was put at different grid points. Its location was calculated by the distance information from two receiving sensor nodes.

To clearly demonstrate capabilities of the ultrasonic ranging system implemented by the developed SOD, the distance errors are labeled in Figure 6 . It is clear that the error is smaller in the center region than in the edge region. This results from the ultrasonic directivity and interference. Based on this experimental result, the maximum location error is $49 \mathrm{~mm}$ within an area of $1 \mathrm{~m}$ by $2 \mathrm{~m}$.

\section{Conclusion}

We have developed a synchronization-on-demand (SOD) protocol for collision-prevention on civil construction sites. To deal with the large area and complicated movement of equipment, instead of full-network synchronization, the proposed SOD protocol can be synchronized within few nodes in $10 \mathrm{~ms}$ with only 0.8 synchronization error. This reduces the wireless communication latency and improves the network stability. In addition, the ultrasonic ranging system with SOD protocol was also experimentally implemented to verify the capability of location identification. Without complicated estimations and calculations, the location error was less than $5 \mathrm{~cm}$ within a $2 \mathrm{~m}$ range. It should be noted that a longer detection range can be achieved by increasing the power of ultrasonic transducers. This work demonstrates a simple and efficient approach to obtain good accuracy, low communication overhead, and robust synchronization for wireless sensor network platforms in automated monitoring at construction sites.

\section{Acknowledgement}

This research was financially supported by National Science Council, Taiwan (Granted Number: NSC 99-2218-E-002-034-; NSC 99-2218-002-017-). 


\section{References}

[1] H. P. Tserng, B. Ran, and J. S. Russell, "Interactive path planning for multi-equipment landfill operations," Automation in Construction, vol. 10, no. 1, pp. 155-168, 2000. doi: 10.1016/S0926-5805(00)00073-X

[2] A. A. Oloufa, M. Ikeda, and H. Oda, "Situational awareness of construction equipment using GPS, wireless and web technologies," Automation in Construction, vol. 12, no. 6, pp. 737-748, 2003. doi: $10.1016 /$ S0926-5805(03)00057-8

[3] W. Wu, H. Yang, D. A. S. Chew, S.-h. Yang, A. G. F. Gibb, and Q. Li, "Towards an autonomous real-time tracking system of near-miss accidents on construction sites," Automation in Construction, vol. 19, no. 2, pp. 134-141, 2010.

doi: 10.1016/j.autcon.2009.11.017

[4] S. Chae and T. Yoshida, "Application of RFID technology to prevention of collision accident with heavy equipment," Automation in Construction, vol. 19, no. 3, pp. 368-374, 2010.

doi: 10.1016/i.autcon.2009.12.008

[5] Y. M. Hsieh and Y. C. Hung, "A scalable IT infrastructure for automated monitoring systems based on the distributed computing technique using simple object access protocol Web-services," Automation in construction, vol. 18 , no. 4, pp. 424-433, 2008.

doi: $10.1016 /$ j.autcon.2008.10.010

[6] A. Milenković, C. Otto, and E. Jovanov, "Wireless sensor networks for personal health monitoring: Issues and an implementation," Computer Communications, vol. 29, no. 13-14, pp. 2521-2533, 2006.

doi: 10.1016/j.comcom.2006.02.011

[7] J. P. Sheu, W. K. Hu, and J. C. Lin, "Ratio-based time synchronization protocol in wireless sensor networks," Telecommunication Systems, vol. 39, no. 1, pp. 25-35, 2008.

doi: $10.1007 / \mathrm{s} 11235-008-9081-5$

[8] B. M. Sadler, "Local and broadcast clock synchronization in a sensor node," IEEE Signal Processing Letters, vol. 13, no. 1, pp. 9-12, 2006. doi: $\underline{10.1109 / \text { LSP.2005.860551 }}$
[9] S. Ganeriwal, C. Pöpper, S. Čapkun, and M. B. Srivastava, "Secure Time Synchronization in Sensor Networks," ACM Transactions on Information and System Security (TISSEC), vol. 11, no. 4, pp. 23:123:35, 2008. doi: $\underline{10.1145 / 1380564.1380571}$

[10] C. H. Rentel and T. Kunz, "A Mutual Network Synchronization Method for Wireless Ad Hoc and Sensor Networks," Mobile Computing, IEEE Transactions on, vol. 7, no. 5, pp. 633-646, 2008. doi: 10.1109/TMC.2007.70787

[11] F. Sivrikaya and B. Yener, "Time synchronization in sensor networks: a survey," IEEE Network, vol. 18, no. 4, pp. 45-50, 2004.

doi: 10.1109/MNET.2004.1316761

[12] K. L. Noh, Q. M. Chaudhari, E. Serpedin, and B. W. Suter, "Novel Clock Phase Offset and Skew Estimation Using Two-Way Timing Message Exchanges for Wireless Sensor Networks," Communications, IEEE Transactions on, vol. 55, no. 4, pp. 766-777, 2007. doi: $10.1109 /$ TCOMM.2007.894102

[13] G. Oriolo, G. Ulivi, and M. Vendittelli, "Fuzzy maps: a new tool for mobile robot perception and planning," Journal of Robotic Systems, vol. 14, no. 3, pp. 179-197, 1997.

[14] IEEE Standard for Information technology-- Local and metropolitan area networks-- Specific requirements-- Part 15.4: Wireless Medium Access Control (MAC) and Physical Layer (PHY) Specifications for Low Rate Wireless Personal Area Networks (WPANs), IEEE Standard 802.15.4, 2006. doi: 10.1109/IEEESTD.2006.232110

[15] "UZ2400 user manual," Uniband Electronic Corporation, 2007.

[16] F. Tong, S. K. Tso, and T. Z. Xu, "A high precision ultrasonic docking system used for automatic guided vehicle," Sensors and Actuators A: Physical, vol. 118, no. 2, pp. 183-189, 2005. doi: $10.1016 /$ j.sna.2004.06.026 